\title{
Topology of neutral hydrogen distribution with the Square Kilometer Array
}

\author{
Yougang Wang ${ }^{1}$, Yidong $\mathrm{Xu}^{1}$, Fengquan $\mathrm{Wu}^{1}$, Xuelei Chen ${ }^{* 1}$, Xin Wang ${ }^{2}$, Juhan \\ $\mathrm{Kim}^{3}$, Changbom Park ${ }^{3}$, Khee-Gan Lee ${ }^{4}$, Renyue Cen ${ }^{4}$ \\ ${ }^{1}$ National Astronomical Observatory, Chinese Academy of Sciences, Beijing, China; \\ ${ }^{2}$ Department of Physics and Astronomy, The Johns Hopkins University, Baltimore, USA; \\ ${ }^{3}$ School of Physics, Korean Institute for Adavanced Studies, Seoul, Korea; \\ ${ }^{4}$ Department of Astrophysical Sciences, Princeton University, Princeton, USA \\ E-mail: wangyg@bao.ac.cn, xuelei@cosmology.bao.ac.cn
}

\begin{abstract}
Morphology of the complex HI gas distribution can be quantified by statistics like the Minkowski functionals, and can provide a way to statistically study the large scale structure in the HI maps both at low redshifts, and during the epoch of reionization (EoR). At low redshifts, the $21 \mathrm{~cm}$ emission traces the underlying matter distribution. Topology of the HI gas distribution, as measured by the genus, could be used as a "standard ruler". This enables the determination of distance-redshift relation and also the discrimination of various models of dark energy and of modified gravity. The topological analysis is also sensitive to certain primordial non-Gaussian features. Compared with two-point statistics, the topological statistics are more robust against the nonlinear gravitational evolution, bias, and redshift-space distortion. The HI intensity map observation naturally avoids the sparse sampling distortion, which is an important systematic in optical galaxy survey. The large cosmic volume accessible to SKA would provide unprecedented accuracy using such a measurement. During the EoR, topology can be a powerful and intuitive tool to distinguish among the different evolutionary stages of reionization, where the ionized regions make up a significant fraction of the volume. Furthermore, it can also discriminate among various reionization models. The genus curves evolve during cosmological reionization, and for different reionization scenarios, the topology of the HI gas distribution can be significantly different even if the global ionization fractions are the same. It can provide clear and intuitive diagnostics for how the reionization takes place, and indirectly probes the properties of radiation-sources. In this brief chapter we will describe the scientific background of the topology study, and forecast the potential of the SKA for measuring cosmological parameters and constraining structure formation mechanism through the study of topology of HI gas distribution.
\end{abstract}

Advancing Astrophysics with the Square Kilometre Array

June 8-13, 2014

Giardini Naxos, Italy

\footnotetext{
*Speaker.
} 


\section{Topology study of HI distribution}

Topology has been introduced in cosmology to describe the properties of the large-scale structure (LSS), and to test the non-Gaussianity of the primordial density fluctuations (Gott et al. 1986; Hamilton et al. 1986; Park et al. 2013). Application of LSS topology study has been extended to measurement of cosmological parameters and constraining galaxy formation mechanism (Park et al. 2005; Choi et al. 2010, 2013). The LSS topology is also a cosmological invariant that can be used to reconstruct the expansion history of the universe (Park \& Kim 2010; Zunckel et al. 2011; Wang et al. 2012; Speare et al. 2013; Blake et al. 2014). Mathematically, geometry of the excursion regions with density above a threshold can be characterized by the Minkowski Functionals (MFs) 1 (Mecke et al. 1994; Pratten \& Munshi 2012; Hikage et al. 2006, 2008). The genus, related to one of the Minkowski functionals, is given by the number of holes minus the number of isolated regions in the iso-density contour surfaces of the smooth density field, and is a measure of topology that quantifies the connectivity of the contour surfaces (Park et al. 2013). All the MFs are analytically known for Gaussian fields, the genus per unit volume as a function of the threshold density $v=\delta / \sigma$ is given by

$$
G(v)=A\left(1-v^{2}\right) e^{-v^{2} / 2}, \quad A=\frac{1}{4 \pi^{2}}\left(\frac{\left\langle k^{2}\right\rangle}{3}\right)^{3 / 2}
$$

The topology of the isodensity contours is insensitive to the systematic effects such as redshiftspace distortion, non-linear evolution, sparse sampling (Park et al. 2005; Park \& Kim 2010). Therefore, any deviation from the Gaussian prediction is evidence for non-Gaussianity, and can constrain the mechanism for primordial non-Gaussianity. The deviation can be caused not just by the three-point function but by all the high-order moments of the density field. Therefore, the study of non-Gaussianity using MFs is complementary to the approach of using three-point correlation function or bispectum, and is in principle able to detect general forms of non-Gaussianity. The Minkowski functionals measured from the $\mathrm{HI} 21 \mathrm{~cm}$ map can characterize the complex distribution of the neutral hydrogen, both in the large-scale structure at low redshifts, and during the epoch of reionization (EoR) at high redshifts.

\subsection{Primordial non-Gaussianity}

The primordial perturbation may deviate from the Gaussian random field during the cosmological phase transition triggered by the spontaneous breakdown of symmetry creating topological defects such as the cosmic monopole, string, wall, and textures. Various non-standard inflationary scenarios which violate the single-field and slow-rolling condition may also produce significant primordial non-Gaussianity. It is usual to parameterize the non-Gaussian features generated by the inflation model as $\Phi(x)=\phi_{G}(x)+f_{\mathrm{NL}}\left[\phi_{G}^{2}(x)-\left\langle\phi_{G}^{2}\right\rangle\right]$ where $\phi_{G}$ is the Gaussian distribution of potential. For this form of primordial non-Gaussianity, the genus curve deviates from the Gaussian

\footnotetext{
${ }^{1}$ In three dimensions, the four Minkowski functionals for the isodensity contours are the volume fraction $V_{0}(v)$, surface area $V_{1}(v)$, mean curvature $V_{2}(v)$ and Euler characteristics $V_{3}(v)=\chi(v)$, which is related to the genus by $\chi=2-2 g$.
} 
model, and up to second order perturbations it is given by Hikage et al. $(2006,2008)$.

$$
\Delta(v) \equiv \delta\left(\frac{G}{A}\right)=-e^{-v^{2} / 2} \times f_{\mathrm{NL}}\left[\left(S_{\mathrm{pri}}^{(1)}-S_{\mathrm{pri}}^{(0)}\right) H_{3}\left(v_{f}\right)+\left(S_{\mathrm{pri}}^{(2)}-S_{\mathrm{pri}}^{(0)}\right) H_{1}(v)\right] \sigma_{0}
$$

where $S_{\text {pri }}^{(a)}$ is the skewness parameter (Matsubara 2003). The measured genus curve can then be used to constrain $f_{N L}$. However, even if the non-Gaussianity can not be parameterized in this form, it would still affect the genus curve, so the topology can be used to probe more general forms of primordial non-Gaussianity.

\subsection{Dark Energy and Modified Gravity}

The LSS topology is relatively stable with the growth of structure. Eq. (1.1) shows that $A$ measures the slope of the power spectrum around the smoothing scale $R_{G}$, and for linear growth $A$ would be conserved (Park et al. 2005), so as long as the same smoothing scale is used, the same comoving volume at different redshifts would enclose nearly the same amount of structures, as illustrated in the left panel of Fig.1 This fact can be used to measure the redshift-distance relation $r(z)$ (Park \& Kim 2010). Since the topology of the structure is not scale-free, the genus enclosed in a wrongly sized volume and smoothed with a wrong scale would lead to deviation from the actual one,

$$
A_{Y}\left(z, R_{G, Y}\right) R_{G, Y}^{3}=A_{X}\left(R_{G, X}\right) R_{G, X}^{3},
$$

where $R$ is the volume, $Y$ is the adopted cosmology parameters while $X$ is the true cosmology. The smoothing scale $R_{G}$ for different cosmologies are related by $\left(R_{G, X} / R_{G, Y}\right)^{3}=\left(D_{A}^{2} / H\right)_{X} /\left(D_{A}^{2} / H\right)_{Y}$, where $D_{A}, H$ are the angular diameter distance and Hubble expansion rate respectively. Utilizing this effect, topology of the large scale structure can serve as a standard ruler in cosmology. This technique has been applied to constrain dark energy model (Blake et al. 2014; Zunckel et al. 2011).

For modified gravity models, the growth rate is different from the general relativity, and in some cases it is scale-dependent. This will induce changes in the genus curve. In the right panel of Fig. 1 the redshift evolution of the genus amplitude for the $f(R)$ theory and some phenomenological modified gravity models are shown. Here the modified gravity model parameter $B_{0}$ is the present day value of the function $B(a)$, which is the square of the Compton scale given by $B(a) \equiv \frac{f_{R R}}{1+f_{R}} R^{\prime} \frac{H}{H^{\prime}}$, where $f_{R}$ and $f_{R R}$ are the first and second derivatives of $f(R)$, with $R$ being the Ricci scalar.

So the topological measurements can also be used to constrain the modified gravity models (Wang et al. 2012). Compared with direct measurement using the growth factor, the topological measurement may be more robust, as they suffer less from the effect of bias, redshift distortion, and non-linearity.

\subsection{Reionization}

The topology analysis provides a direct and sensitive probe for the detailed process of cosmic reionization (Lee et al. 2008; Hong et al. 2014). Using the genus of HI density contours as a quantitative measure of topology, the reionization process of the intergalactic medium (IGM) can be divided into four distinct topological phases for the standard scenario of reionization: (1) pre-reionization, before the formation of any HII bubbles, the HI distribution reflects the primodial 

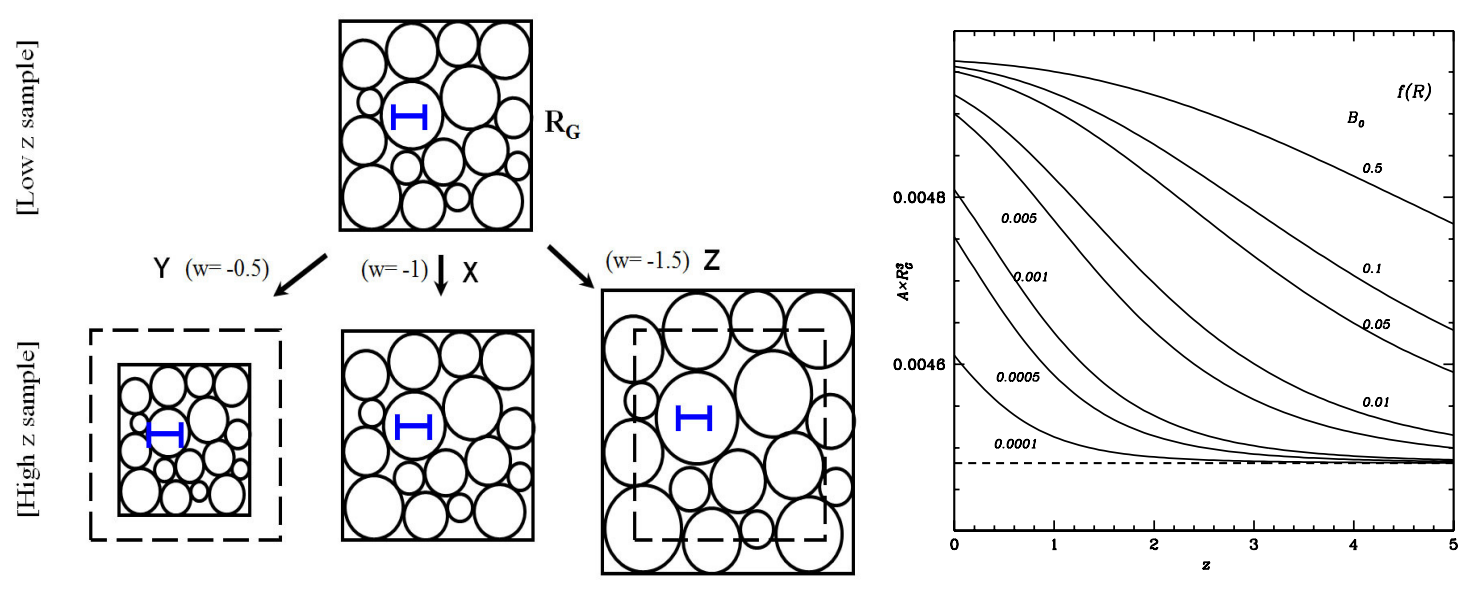

Figure 1: Left: The number of structures as cosmic standard ruler. Right: the redshift evolution of genus amplitude for $f(R)$ and phenomenological modified gravity models with different $B_{0}$ values.

density fluctuations, the genus curve is consistent with a Gaussian density distribution, and topology remains unchanged as the HI density evolves linearly; (2) pre-overlap, characterized by a topology dominated by isolated HII bubbles. At the earlier stages of this phase, the number of HII bubbles increases gradually with time, resulting in an increase in the amplitude of the genus curve, while at the later stages when HII bubbles start to merge, the genus curve goes through a turnover and subsequently decreases in amplitude. (3) overlap, during which the amplitude of the genus curve drops significantly as the HII bubbles rapidly merge; (4) postoverlap, when the IGM is almost ionized, and the evolution of the genus curve is consistent with an decreasing number of isolated neutral islands. The measurement of the genus curve can be used to characterize the evolutionary stages of the neutral topology and to identify the redshifts at which the different stages of reionization occur, thus distinguishing different reionization scenarios. This in turn provides information on the nature and properties of the early luminous sources.

\section{The SKA performance}

The observation of HI emission is presently limited to $z<0.2$ by the sensitivity of existing telescopes. The SKA, with its great increase in sensitivity, will allow us to observe the HI at much higher redshifts, and probe the topology of HI distribution with unprecedented capability. The SKA will be built in two stages (Dewdney et al. 2013; Braun), the SKA-1 will consist of three arrays: SKA1-mid, SKA1-sur and SKA1-low, the features are listed in Table.1; The SKA2 is to be designed in the future, but should be about 10 times larger in mid-frequency and 4 times larger in the low frequencies.

The SKA1-low is designed for observation of the high redshifts of the EoR, while SKA1-mid for the low-to-mid-redshifts of post-reionization Universe, and SKA1-sur with its larger FoV is suitable for survey of large area. All of these can be used for HI topology study. The topological analysis can be applied on data from all HI surveys. We expect that large HI surveys will be one of 
Table 1: The three SKA1 arrays.

\begin{tabular}{|c|c|c|}
\hline \hline Array & configuration & relevant band \\
\hline SKA1-mid & $190 \times 15 \mathrm{~m}$ dish +64 MeerKAT 13.5m dish & band 1 $(0.35-1.05 \mathrm{GHz})$, band 2(0.95-1.76GHz) \\
\hline SKA1-sur & $60 \times 15 \mathrm{~m}$ dish +36 ASKAP 12m dish (PAF) & band 1 $(0.35-0.5)$ and band 2 $0.65-1.67 \mathrm{GHz}$. \\
\hline SKA1-low & 250,000 log periodic antenna in 866 stations & $50-350 \mathrm{MHz}$ \\
\hline \hline
\end{tabular}

the key science projects of SKA, being carried out with multiple applications, and the HI topology can be studied in conjunction with the other projects.

A classic approach is the HI galaxy survey where the individual galaxies are observed as objects whose HI content exceeds the detection threshold:

$$
S_{\text {lim }}=N_{t h} \frac{k T_{\text {sys }}}{A_{\text {eff }} \sqrt{\Delta v t}}=N_{t h} \frac{\mathrm{SEFD}}{\sqrt{\Delta v t}}
$$

where $N_{t h}$ is a preset threshold value, e.g. 5 or 10 . The SKA is potentially capable of surveying a billion of galaxies, with SKA-1 it is capable of surveying $10^{7-8}$ galaxies(Abdalla \& Rawlings 2005; Myers et al. 2009) at $z$ up to $\sim 1.5$. But even smaller surveys, e.g. those conducted with SKA-1 early science, or for small deep field, would for the first time reveal the HI distribution at redshifts much beyond our current limit, and the topological analysis will help us to understand quantitatively the characteristics of $\mathrm{HI}$ distribution and redshift evolution, as has been down for low redshift optical galaxies (Choi et al. 2010, 2013).

Another approach is intensity mapping (IM): the sky is mapped at low angular resolution, such that individual galaxies can not be distinguished, but data of HI large scale distribution can still be obtained (Chang et al. 2008). For the EoR experiments, including that of the SKA-low, due to the low angular resolution, this will also be the mode of observation. A few dedicated experiments, such as the $\mathrm{CHIME}^{2}$ and Tianlai ${ }^{3}$ are designed to conduct IM observations. While the SKA1-mid or SKA1-sur are not designed for this, it has been proposed that IM surveys may be conducted with the dishes used individually with output autocorrelation, while being calibrated using the interferometry (Santos 2015). Whether this would work still needs to be tested in the field, but if successful, it would allow higher sensitivity on interested scales at the price of lower angular resolution.

In Table2 we list both the HI galaxy and IM survey parameters we adopted for our forecast on dark energy and modified gravity. For SKA-low, as the design is still very uncertain, we do not make a full forecast here, but only discuss the potential of the measurement.

\section{Large Scale Structures}

After EoR, with the IGM highly ionized, the neutral hydrogen resides mainly in galaxies, and the HI topology traces out the LSS. We can use this observation to constrain the primordial non-Gaussianity, dark energy, and modified gravity.

\footnotetext{
${ }^{2} \mathrm{http}: / /$ chime.phas.ubc.ca/

${ }^{3}$ http://tianlai.bao.ac.cn/
} 
Table 2: SKA survey parameters utilized in this paper.

\begin{tabular}{|c|c|c|c|}
\hline galaxies surveys & survey area $\left(\mathrm{deg}^{2}\right)$ & integration time $(h)$ & flux limit $(\mathrm{mJy})$ \\
\hline SKA1-Mid & $1,000(5,000)$ & $5,000(25,000)$ & 0.13 \\
SKA2-Mid & $1,000(5,000)$ & $5,000(25,000)$ & 0.05 \\
SKA1-Sur & 30,000 & $7,500(50,000)$ & $0.4(0.15)$ \\
SKA2-Sur & 30,000 & 7,500 & 0.05 \\
\hline \hline HI intensity mapping & survey area $\left(\mathrm{deg}^{2}\right)$ & integration time $(h)$ & frequency band $(\mathrm{MHz})$ \\
\hline SKA1-Mid-SD & 30,000 & 10,000 & $350-1050$ \\
SKA1-Mid-Int & 30,000 & 10,000 & $350-800$ \\
SKA2-Mid-Int & 30,000 & 10,000 & $350-950$ \\
\hline
\end{tabular}

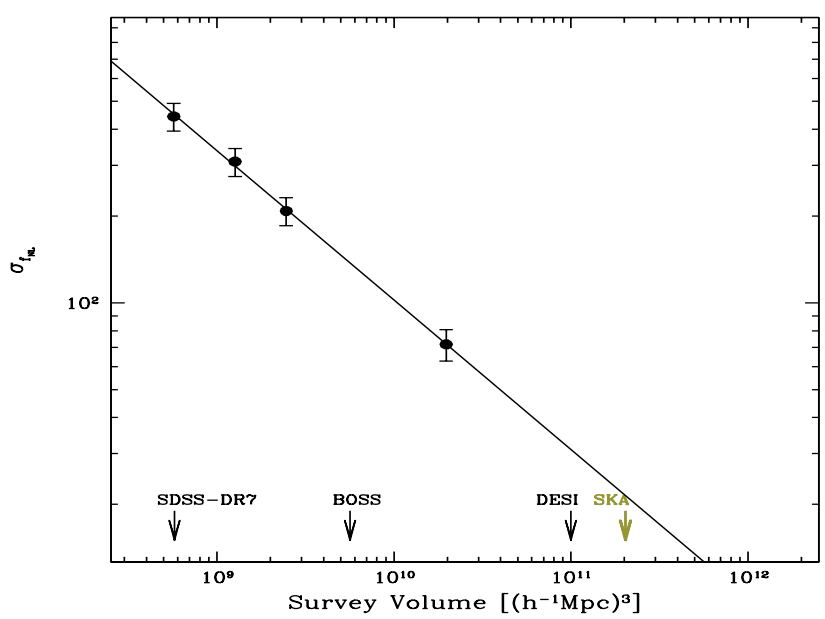

Figure 2: The measurement error of $f_{\mathrm{NL}}$ from genus curves. We mark the survey volumes of previous (SDSS-DR7, BOSS) and forth-coming (DESI \& SKA) surveys.

For primordial non-Gaussianity, Fig. 2 shows the $f_{\mathrm{NL}}$ constraints from measurement of genus curve in the Horizon Run III simulation, which has a volume of $10.8^{3} h^{-3} \mathrm{Gpc}^{3}$. We employed a Gaussian smoothing of $R_{G}=22 h^{-1} \mathrm{Mpc}$ to build a density field from subcubes, and compare the result for four survey volumes: SDSS, BOSS, DES and SKA. If the SKA-2 will observe galaxy distribution out to $z=3$ with a solid angle of $20,000 \mathrm{deg}^{2}$, then using the genus-curve we can measure $f_{\mathrm{NL}}$ with an error, $\sigma\left(f_{\mathrm{NL}}\right)=20$. We should note that this is comparable to the CMB limits, and the genus measurement can detect more general forms of non-Gaussianity.

Using the genus curve as standard ruler, dark energy equation of state parameters $w_{0}, w_{a}$ can be constrained. As shown in Table (2), we consider a relatively deep survey using SKA1-Mid compared with shallow but wider surveys for SKA1-Sur.

The HI galaxies survey constraints are plotted in Fig. (3), and IM survey constraints are plotted in the right panel of Fig.4. With a conservative assumption of the survey parameters that require 

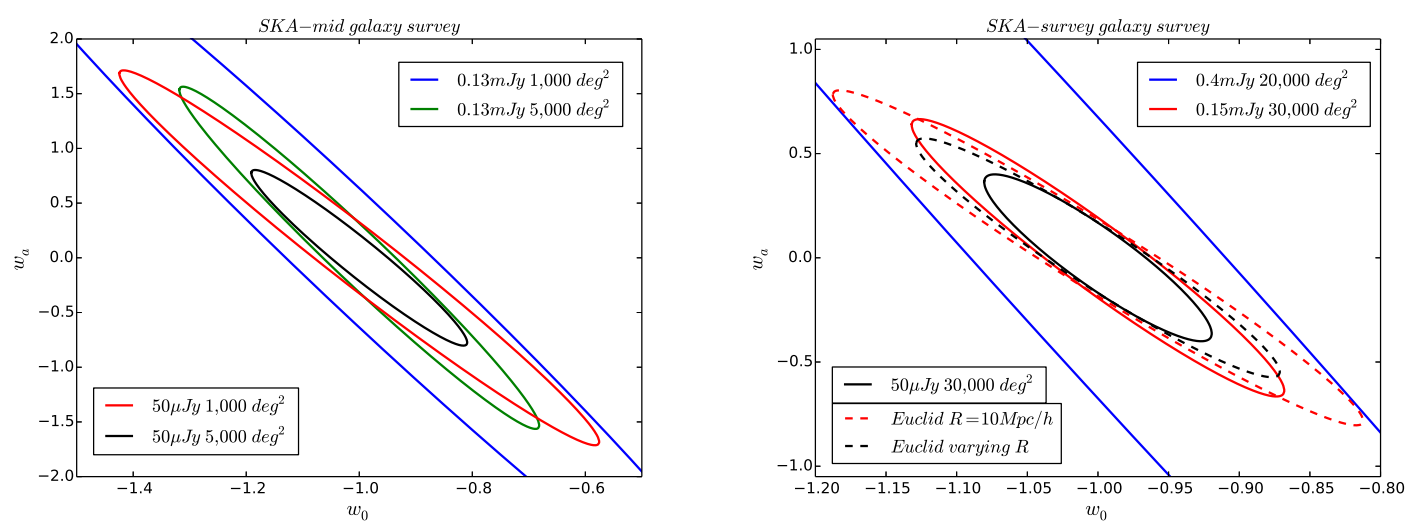

Figure 3: Constraint on dark energy with HI galaxy survey. Left: HI galaxy survey with SKA1-mid, Right: HI galaxy survey with SKA1-survey. The dashed contours indicate the constraints from Euclid.
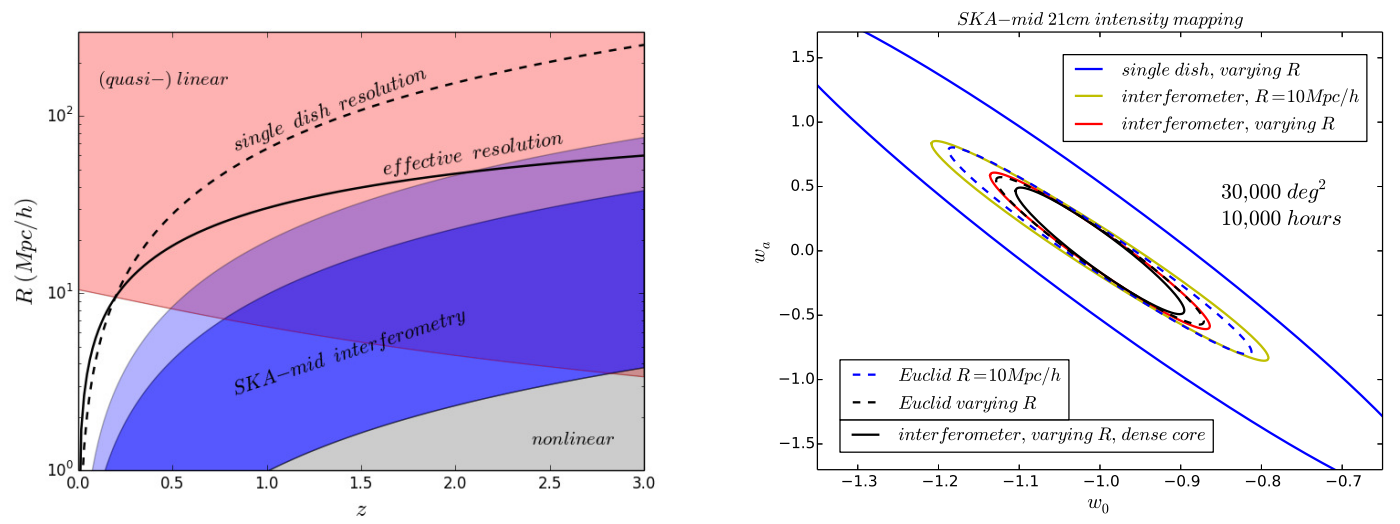

Figure 4: SKA-mid HI intensity mapping. Left: Various comoving scales as a function of redshift. The upper red region denotes the quasi-linear regime where the systematics of genus statistics are well-controlled. Blue bands are most sensitive scales that would be observed by the core of SKA-mid interferometer, where we assume the baseline range from $100 \mathrm{~m}$ ( $50 \mathrm{~m}$ for lighter blue band) to $1000 \mathrm{~m}$. The grey region at bottomright corner are scales available with the SKA spiral arm baselines. The black dashed line indicates the physical angular resolution of single dish. The solid black line is the effective resolution $\left(R_{\text {ang }}^{2} R_{\text {freq }}\right)^{1 / 3}$, where we utilize the minimum quasilinear scale, i.e. the boundary of red region, as $R_{\text {freq }}$. Right: Constraints on dark energy equation of state $w_{0}$ and $w_{a}$. In the calculation, 'varying R' means the smoothing length is selected using smallest quasi-linear scale. The dashed contours indicate the constraints from Euclid. 
5,000 hours integration time, neither constraint is very stringent for SKA1, but it is independent of other techniques, and strong constraints can be achieved with SKA2. As we already know that BAO gives better constraints than genus for dark energy, we just give the constraints of our calculation, and refer the interested reader to the chapter written by Bull et al. (this edition).

For the IM, there is certain complications. Unlike the BAO technique, where the physical scale is fixed, the topological standard ruler measure the scale around the smoothing length. To achieve better statistics, smaller smoothing length is desirable, though the systematics such as nonlinear growth put limits on the usable scale. In the left panel of Fig.4), we illustrate various physical scales for SKA1-mid. The quasi-linear region where systematics is well controlled is indicated by the upper red region.

For the single dish observing mode, the angular resolution by the $15 \mathrm{~m}$ dish is poor (black dashed-line), varying from $30 \mathrm{Mpc} / h$ at $z=0.5$ to above $200 \mathrm{Mpc} / h$ at $z=3$. However, in the line-of-sight direction the resolution is good, the effective $R_{\text {eff }}$,

$$
R_{\text {eff }}=\left(R_{\text {ang }}^{2} R_{\text {freq }}\right)^{1 / 3}
$$

becomes much smaller. Assuming a reasonable $R_{\text {freq }}$ as indicated by the lower edge of the red region, the effective smoothing length varies from $10 \mathrm{Mpc} / h$ to $60 \mathrm{Mpc} / h$. The cosmological constraints of this observation mode is shown as the blue contour in the right panel.

If sufficient number of short baselines is available, it may be possible to make an IM survey in the interferometric mode. We assume a compact core in the center of SKAmid, the blue belt in the left panel illustrates the most sensitive physical scale of this core, where we assume baselines distributed from $100 \mathrm{~m}$ (50m for lighter blue region) to $1000 \mathrm{~m}$. With the systematic limit from nonlinear scale, one would be able to observe higher redshift volume from $z=0.78(z=0.5)$ at much smaller smoothing length and therefore a better statistical accuracy. As shown in the right panel, such observation mode improve the constraints significantly.

For modified gravity models, we consider specifically the $f(R)$ theory with a single model parameter $B_{0}$ characterizing the present value of Compton scale. HI galaxy survey by SKA2 will be able to constrain $B_{0}$ to $5 \times 10^{-5}$ at $1-\sigma$ level for a fiducial value of $B_{0}=10^{-4}$, while the best constraints from intensity mapping is $8 \times 10^{-5}$. This is much better than the current strongest constraints of $1.1 \times 10^{-3}$ at $95 \%$ C.L..

\section{Reionization}

The genus curve can be used to distinguish the different phases of reionization (Lee et al. 2008; Hong et al. 2014) discussed in §1.3. In Fig. 5 we show the genus curve from an EoR simulation (Trac et al. 2008), for four redshifts during the EoR. As expected, at the highest $\operatorname{redshift}(z=$ $19.7, x_{i} \approx 0$ ), the genus curve has the shape for that of a Gaussian distribution. The amplitude of the curve increases in the pre-overlap phase $\left(z=9, x_{i}=0.14\right)$, but retains the shape for Gaussian distribution. The shape of the genus curve is drastically different during the overlap phase $\left(z=6.97, x_{i}=0.65\right)$. Finally, the curve returns to the shape of Gaussian distribution in the postreionization phase $\left(z=5.99, x_{i}=0.99\right)$. From Eq.1.1 we see the amplitude of genus curve $A \propto k_{e}^{3}$, $k_{e}$ first increases as the HII bubbles grows in number, and then decreases as the bubbles merge and overlap. Measuring $k_{e}$ from $A$ can provide information on the bubble sizes. 


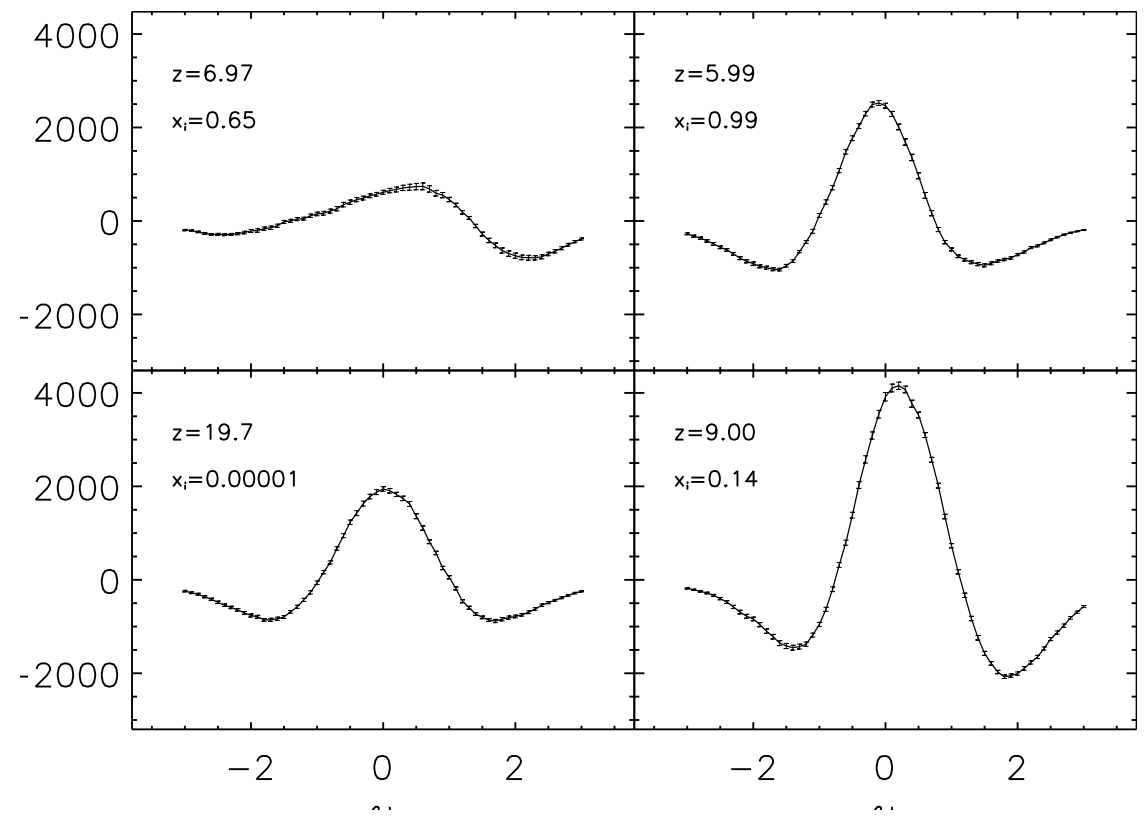

Figure 5: Genus curve and statistical error of $\delta T_{b}$ at different redshifts from a simulation (Trac et al. 2008), the simulation box has a side length of $100 \mathrm{Mpc} \mathrm{h}^{-1}$, and smoothing length is $1 \mathrm{Mpc} \mathrm{h}^{-1}$

These curves are obtained with a smoothing length of $1 \mathrm{Mpc} / h$. Along with the genus curves, the statstical error obtained from a $100 \mathrm{Mpc} / h$ simulation box is plotted. The design of the SKAlow (Dewdney et al. 2013; Braun 2013) is still very uncertain, but it is agreed that there should be a compact core within $1 \mathrm{~km}$ which provides high sensitivity observation on scales of a few arcmin for redshift 10. This is roughly sufficient for the measurement. The observation of SKA1-low for a deep field should be able to yield data which can be compared with such simulations.

\section{Conclusion}

Topology can be very useful at characterizing random fields. The SKA, with its high sensitivity, can observe the HI distribution much beyond the current limits. We can apply the topological analysis method to the SKA HI survey data. Such analysis can also be used to test or constrain primordial non-Gaussianity, dark energy and modified gravity. The constraints for the model parameters from the topological method are not as tight as the traditional methods, e.g., dark energy constraints from BAO measurements (See Bull et al. chapter) and primordial non-Gaussianity from scale-dependent bias and bispectrum measurements (See Camera et al. chapter), but it is very robust against non-linearity, bias and redshift distortion in the evolution of large scale structure. During the EoR, it can also distinguish different evolution phases and characters of reionization model. We made forecasts for some of these applications, in both HI galaxy survey and HI intensity mapping survey, and obtained constraints for different survey parameters. For SKA1, the precision of the measurement with the genus curve is moderate, but we anticipate that the addition of other Minkowski functionals would help improve these constraints, and for SKA2 the result is competitive. More importantly, the genus curve is capable of probing non-Gaussian features which 
are not parameterized in the standard form, so with SKA it would be an important tool for probing and discovering non-Gaussianity in unexplored regimes.

\section{References}

Abdalla, F. B. \& Rawlings, S. 2005, MNRAS, 360, 27

Blake, C., James, J. B., \& Poole, G. B. 2014, MNRAS, 437, 2488

Chang, T.-C., Pen, U.-L., Peterson, J. B., \& McDonald, P. 2008, Physical Review Letters, 100, 091303

Choi, Y.-Y., Kim, J., Rossi, G., Kim, S. S., \& Lee, J.-E. 2013, ApJS, 209, 19

Choi, Y.-Y., Park, C., Kim, J., et al. 2010, ApJS, 190, 181

Gott, III, J. R., Dickinson, M., \& Melott, A. L. 1986, ApJ, 306, 341

Hamilton, A. J. S., Gott, III, J. R., \& Weinberg, D. 1986, ApJ, 309, 1

Hikage, C., Coles, P., Grossi, M., et al. 2008, MNRAS, 385, 1613

Hikage, C., Komatsu, E., \& Matsubara, T. 2006, ApJ, 653, 11

Hong, S. E., Ahn, K., Park, C., et al. 2014, Journal of Korean Astronomical Society, 47, 49

Lee, K.-G., Cen, R., Gott, III, J. R., \& Trac, H. 2008, ApJ, 675, 8

Matsubara, T. 2003, ApJ, 584, 1

Mecke, K. R., Buchert, T., \& Wagner, H. 1994, A\&A, 288, 697

Myers, S. T., Abdalla, F. B., Blake, C., et al. 2009, in ArXiv Astrophysics e-prints, Vol. 2010, astro2010: The Astronomy and Astrophysics Decadal Survey, 219

Park, C., Kim, J., \& Gott, III, J. R. 2005, ApJ, 633, 1

Park, C. \& Kim, Y.-R. 2010, ApJ, 715, L185

Park, C., Pranav, P., Chingangbam, P., et al. 2013, Journal of Korean Astronomical Society, 46, 125

Pratten, G. \& Munshi, D. 2012, MNRAS, 423, 3209

Speare, R., Gott, J. R., Kim, J., \& Park, C. 2013, ArXiv e-prints

Trac, H., Cen, R., \& Loeb, A. 2008, ApJ, 689, L81

Wang, X., Chen, X., \& Park, C. 2012, ApJ, 747, 48

Zunckel, C., Gott, J. R., \& Lunnan, R. 2011, MNRAS, 412, 1401 